\title{
Status and prospects of the BaBar SVT
}

V. Re ${ }^{\mathrm{a}}$, M. Bruinsma ${ }^{\mathrm{b}, *}$, S. Curry ${ }^{\mathrm{b}}$, D. Kirkby ${ }^{\mathrm{b}}$, J. Berryhill $^{\mathrm{c}}$, S. Burke ${ }^{\mathrm{c}}$, D. Callahan ${ }^{\mathrm{c}}$, C. Campagnaric ${ }^{\mathrm{c}}$ B. Dahmes ${ }^{\mathrm{c}}$, D. Hale ${ }^{\mathrm{c}}$, P. Hart ${ }^{\mathrm{c}}$, S. Kyre ${ }^{\mathrm{c}}$, S. Levy ${ }^{\mathrm{c}}$, O. Long ${ }^{\mathrm{c}}$, M. Mazur ${ }^{\mathrm{c}}$, J. Richman ${ }^{\text {c }}$ J. Stoner ${ }^{c}$, W. Verkerke ${ }^{c}$, T. Beck ${ }^{\mathrm{d}}$, A.M. Eisner ${ }^{\mathrm{d}}$, J. Kroseberg $^{\mathrm{d}}$, W.S. Lockman ${ }^{d}$, G. Nesom ${ }^{d}$, A. Seiden ${ }^{d}$, P. Spradlin ${ }^{d}$, W. Walkowiak ${ }^{d}$, M. Wilson ${ }^{d}$,

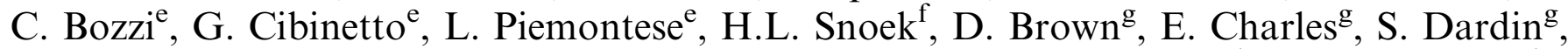
F. Goozen ${ }^{\mathrm{g}}$, L.T. Kerth ${ }^{\mathrm{g}}$, A. Gritsan ${ }^{\mathrm{g}}$, G. Lynch $^{\mathrm{g}}$, N.A. Roe ${ }^{\mathrm{g}}$, C. Chen ${ }^{\mathrm{h}}$, W. Hulsbergen ${ }^{\mathrm{h}}$, C.K. Lae ${ }^{\mathrm{h}}$, V. Lillard ${ }^{\mathrm{h}}$, D. Roberts ${ }^{\mathrm{h}}$, A. Lazzaro ${ }^{\mathrm{i}}$, F. Palombo ${ }^{\mathrm{i}}$, L. Ratti ${ }^{\mathrm{j}}$, P.F. Manfredi ${ }^{\mathrm{j}}$, E. Mandellij, C. Angelini ${ }^{\mathrm{k}}$, G. Batignani ${ }^{\mathrm{k}}$, S. Bettarini ${ }^{\mathrm{k}}$, M. Bondioli ${ }^{\mathrm{k}}, \mathrm{F}_{\text {. Bosi }}{ }^{\mathrm{k}}, \mathrm{F}^{\mathrm{j}}$ Bucci $^{\mathrm{k}}$, G. Calderini ${ }^{\mathrm{k}}$, M. Carpinellik ${ }^{\mathrm{k}}$, M. Ceccanti ${ }^{\mathrm{k}}$, F. Forti ${ }^{\mathrm{k}}$, M.A. Giorgi ${ }^{\mathrm{k}}$, A. Lusiani ${ }^{\mathrm{k}}$, P. Mammini ${ }^{\mathrm{k}}$, G. Marchiori ${ }^{\mathrm{k}}$, M. Morganti ${ }^{\mathrm{k}}$, F. Morsani ${ }^{\mathrm{k}}$, N. Neri ${ }^{\mathrm{k}}$, E. Paoloni ${ }^{\mathrm{k}}$, A. Profeti ${ }^{\mathrm{k}}$, M. Rama ${ }^{\mathrm{k}}$, G. Rizzo ${ }^{\mathrm{k}}$, G. Simi ${ }^{\mathrm{k}, \mathrm{l}}$, J. Walsh $^{\mathrm{k}}$, P. Elmer ${ }^{\mathrm{m}}$, A. Perazzo ${ }^{1}$, P. Burchat ${ }^{\mathrm{n}}$,

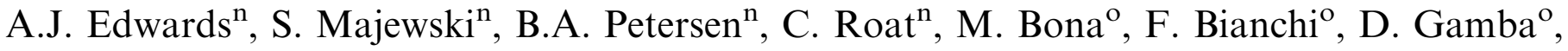

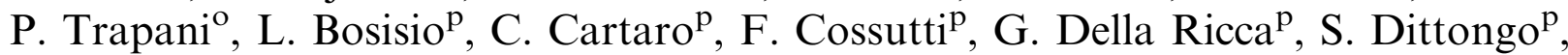
S. Grancagnolo ${ }^{\mathrm{p}}$, L. Lanceri ${ }^{\mathrm{p}}$, L. Vitale ${ }^{\mathrm{p}}$, M. Datta ${ }^{\mathrm{q}}$, A. Mihalyi ${ }^{\mathrm{q}}$

\author{
${ }^{\mathrm{a}}$ INFN-Pavia and Università di Bergamo, Italy \\ ${ }^{\mathrm{b}}$ University of California, Irvine, CA, USA \\ ${ }^{\mathrm{c}}$ University of California, Santa Barbara, CA, USA \\ ${ }^{\mathrm{d}}$ University of California, Santa Cruz, USA \\ ${ }^{\mathrm{e}} I N F N$-Ferrara and Università di Ferrara, Italy \\ ${ }^{\mathrm{f}}$ NIKHEF, Amsterdam, Netherland \\ ${ }^{\mathrm{g}}$ Lawrence Berkeley National Laboratory, Berkeley, CA, USA \\ ${ }^{\mathrm{h}}$ University of Maryland, MD, USA \\ ${ }^{\mathrm{i}} I N F N$-Milano and Università di Milano, Italy \\ ${ }^{\mathrm{j}} I N F N$-Pavia and Università di Pavia, Italy \\ ${ }^{\mathrm{k}}$ INFN-Pisa and Università di Pisa, Italy \\ ${ }^{1}$ Stanford Linear Accelerator Center, Stanford, CA, USA \\ ${ }^{\mathrm{m}}$ Princeton University, USA \\ ${ }^{\mathrm{n}}$ Stanford University, Stanford, CA, USA \\ ${ }^{\circ} I N F N$-Torino and Università di Torino, Italy \\ ${ }^{\mathrm{p}}$ INFN-Trieste and Università di Trieste, Italy \\ ${ }^{\mathrm{q}}$ University of Wisconsin, Madison, USA \\ Available online 19 December 2005
}

\section{Abstract}

The BABAR Silicon Vertex Tracker (SVT) has been efficiently operated for five years since the start of data taking in 1999. It has met design requirements and no degradation in its performance has been observed thus far. However, because of higher than expected

\footnotetext{
*Corresponding author.

E-mail address: bruinsma@slac.stanford.edu (M. Bruinsma).
} 
background levels, and anticipated further increases in luminosity and dose rates, we have done a thorough study to assess the viability of operating the SVT until the end of the decade.

\section{The $B_{A} B_{A} R$ silicon vertex tracker}

The $\mathrm{BABAR}$ experiment [1] is dedicated to a systematic study of $\mathrm{CP}$ asymmetries in $B$ decays and to precision measurements of the CKM quark mixing matrix. It has thus far recorded over 200 million $B \bar{B}$ pairs produced in collisions of a $3.1 \mathrm{GeV}$ positron beam and a $9.0 \mathrm{GeV}$ electron beam. The beams provide a center-of-mass energy of $10.58 \mathrm{GeV}$, near the $r(4 S)$ resonance, with a net boost that gives a measurable separation of the two $B$ decays.

The $\mathrm{BABAR}$ SVT is the sub-detector that is nearest to the $e^{+} e^{-}$interaction point. Its primary purpose is a precise determination of track parameters of charged particles to allow the reconstruction of vertices with a resolution that is sufficient to disentangle the two $B$ decays. They have an average separation along the beam axis $(z)$ of $280 \mu \mathrm{m}$ and the achieved vertex resolution in $z$ is $80 \mu \mathrm{m}$. The SVT also enables the reconstruction of low-momentum $\left(p_{T}<\right.$ $120 \mathrm{MeV}$ ) charged particles, in particular slow pions from $D^{*}$ decays, that do not fully traverse the main $\mathrm{BABAR}$ tracker.

The SVT, described in greater detail elsewhere [2], is a silicon micro-strip detector comprising over 140000 channels on 340 double-sided, AC-coupled silicon wafers arranged in five layers. The first layer is as close as $3.2 \mathrm{~cm}$ to the beam axis, while the last layer is $14.0 \mathrm{~cm}$ away. Each wafer has strips oriented perpendicular (' $z$-strips') and parallel (' $\phi$-strips') to the beam axis on either side, and the readout pitch ranges from $50 \mu \mathrm{m}$ for the $\phi$-strips in the first layer to $210 \mu \mathrm{m}$ for the $z$-strips in the outermost layers. The entire detector is immersed in a $1.5 \mathrm{~T}$ magnetic field.

The strip signals are digitized using custom built frontend chips called AToM [3], each of which serves 128 channels in parallel. The digitization pipeline of a single channel on the AToM has an analog front end (preamplifier, shaper and a comparator with programmable threshold) and a 193-wide circular buffer, which is clocked at $15 \mathrm{MHz}$ and provides a maximum trigger latency of $12.7 \mu \mathrm{s}$. Upon the reception of a L1-trigger, the AToM searches for a hit within a $1 \mu$ s window of the buffer, and sums up the number of consecutive hits. This number measures the 'Time-Over-Threshold', which is proportional to the logarithm of the collected charge.

\section{Performance}

Of the 208 readout sections of the SVT, nine were inoperable from the start of data taking in 1999. Five of these failures were caused by bad connections and recovered during an access to the detector in 2002. In addition, approximately $2 \%$ of the individual channels were unbonded or otherwise unresponsive. The $5 \%$ missing channels do not significantly affect the overall performance of the SVT, thanks to a sufficient redundancy in the detector design.

The hit efficiency, measured using reference tracks passing the active area, averages at $97 \%$, when the four dead readout sections are discounted. The efficiency for reconstructing low momentum particles is $75 \%$ for momenta above $100 \mathrm{MeV}$ and $90 \%$ for momentum above $200 \mathrm{MeV}$. The hit resolution is measured using highmomentum particles in two-prong events. For particles incident on the wafer under a straight angle, the hit resolution is between 10 and $15 \mu \mathrm{m}$ for the three innermost layers, and between 30 and $40 \mu \mathrm{m}$ for the two outermost layers. This gives a vertex resolution in for fully reconstructed decays of better than $80 \mu \mathrm{m}$ in $z$. The resolution in the separation of the vertices of a fully reconstructed decay and an inclusively reconstructed decay, relevant for most of the time-dependent CP analyses in BABAR, is $190 \mu \mathrm{m}$, dominated by the uncertainty in the inclusively reconstructed (tag side) vertex. No loss of efficiency or decrease in resolution has been observed in the first five years of operation.

\section{Radiation monitoring}

To prevent excessive radiation damage to the SVT, a radiation monitoring and protection system (SVTRAD [4]) triggers beam aborts whenever background radiation levels are anomalously high. An abort decision is issued within $1 \mathrm{~ms}$ when dose rates higher than $1 \mathrm{Rad} / \mathrm{ms}$ are observed in order to prevent acute damage. Furthermore, a long-term radiation budget is enforced by triggering a beam abort when the dose rate persistently $(10 \mathrm{~min})$ exceeds $50 \mathrm{mrad} / \mathrm{s}$, more than twice the level observed during normal running conditions. Much of the background radiation is concentrated in the horizontal plane, where off-momentum beam particles are swept into the detector by the final-focusing dipole magnet.

Radiation levels have been higher than anticipated: some parts of the detector have already accumulated over $2 \mathrm{MRad}$, a level not expected to be reached over the full lifetime of the experiment. While the PEP-II accelerator has already delivered instantaneous luminosities of over $9 \times 10^{33} \mathrm{~cm}^{-2} \mathrm{~s}^{-1}$ (over three times the design value), 


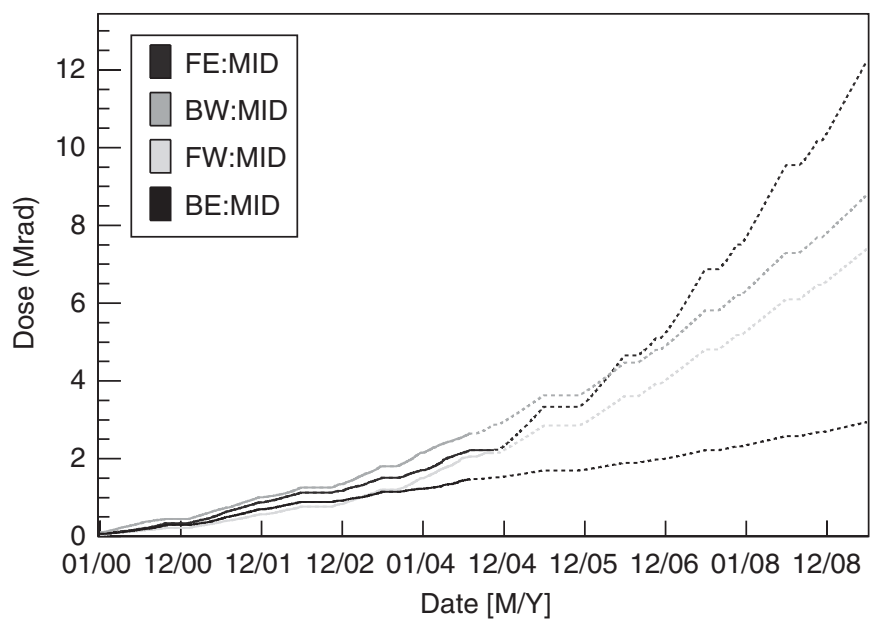

Fig. 1. Measured (solid) and predicted (dashed) radiation doses in four locations in the horizontal (MID) plane. The labels refer to Forward(F)/ Backward(B) and East(E)/West(W), where Forward(Backward) is approximately $10 \mathrm{~cm}$ upstream(downstream) the interaction point and East(West) is $3 \mathrm{~cm}$ inside(outside) the circular beam line.

Table 1

Potential sources of damage to the SVT due to acute or chronic radiation levels

\begin{tabular}{lll}
\hline & Wafers & Readout electronics \\
\hline Acute & p-stop shorts & \\
& coupling capacitor breakdown & \\
Chronic & Leakage current increase & noise increase \\
& shift in depletion voltage & gain decrease \\
& loss of charge coll. efficiency & pedestal shift \\
& & \\
& & \\
\end{tabular}

further increases are planned. Dose rates are expected to increase correspondingly, posing a potentially critical problem for the SVT in the future. Fig. 1 shows the accumulated dose and the dose expected until the end of running in 2009.

\section{Radiation damage studies}

The SVT group has performed extensive tests to assess the viability of running the SVT until 2009. The potential degradation due to any of the mechanisms listed in Table 1 has been evaluated in a series of dedicated radiation tests at Elettra [5], LBNL and SLAC.

\subsection{Damage to the wafers}

Bulk damage to the silicon wafer causes a loss of charge collection efficiency (CCE), an increase in leakage current and corresponding shot noise and a shift in depletion voltage. To determine the magnitude of these effects, $\mathrm{Si}$ wafers equipped with readout electronics were irradiated with the $0.9 \mathrm{GeV}$ electron beam of Elettra in six steps up to a total dose of $9 \mathrm{MRad}$ [6]. After each irradiation step, the loss in CCE was measured using a LED with $\lambda=1060 \mathrm{~nm}$. The depletion voltage was determined by measuring the CCE and the inter-strip capacitance as a function of the bias voltage. A loss in CCE of about $1 \%$ /MRad was observed. The depletion voltage steadily dropped until type inversion at $3 \mathrm{MRad}$, in agreement with the NIEL hypothesis, and rose to $70 \mathrm{~V}$ at $9 \mathrm{MRad}$. This indicates that we will be able to fully deplete the detector until the end of the decade. The increase in leakage current of $0.5-0.7 \mu \mathrm{A} \mathrm{MRad}^{-1} \mathrm{~cm}^{-2}$ at $17^{\circ} \mathrm{C}$ is in agreement with observations of the installed SVT and shows shot noise to be a negligible problem.

\subsection{Damage to the front-end electronics}

A set of AToM chips connected to a half module was irradiated up to $5 \mathrm{MRad}$ with ${ }^{60} \mathrm{Co}$ sources at SLAC and LBNL and up to $9 \mathrm{MRad}$ with the $0.9 \mathrm{GeV}$ electron beam of Elettra [7]. The chips were powered and clocked during irradiation and showed no digital failures. An increase of noise of $15-20 \% / \mathrm{MRad}$ and a decrease in gain of $4 \%$ / MRad was observed, consistent with observations on the installed modules.

The installed modules showed an unexpected rise in the comparator pedestals after an accumulated dose of $1 \mathrm{MRad}$. After rising to approximately half of the dynamic range at $1.5 \mathrm{MRad}$, the pedestals slowly decreased again to their nominal levels at $3 \mathrm{MRad}$. This effect has been confirmed in the lab tests and is attributed to an asymmetry in the comparator circuitry of the AToM. The affected modules can still be operated by regularly adjusting the programmable threshold values.

\subsection{Expected impact on performance}

As shown in Fig. 2, the noise increase on the AToM chips is the dominant effect in the reduction of the signalto-noise $(S / N)$ ratio due to radiation damage. We expect a significant impact on SVT performance at $S / N$ ratios lower than 10 , reached after an accumulated dose of approximately $6 \mathrm{MRad}$. We expect this dose within the next two years for some of the chips in horizontal plane (see Fig. 1) of the first two layers.

In view of our expectation that some parts of the SVT will become inoperable, detailed studies have been performed to quantify the possible impact on physics analyses. In a set of scenarios ranging from realistic to pessimistic, we find a loss in the reconstruction efficiency of $2-4 \%$ for $B^{0} \rightarrow J / \psi K_{S}^{0}$ decays and $2-10 \%$ for slow pions. No significant impact on the mass or vertex resolution has been observed. We judge the loss in efficiency to be acceptable and therefore do no plan to replace affected modules.

The foreseen increase in background levels will also increase the hit occupancy during normal running. This can jeopardize the event reconstruction with a loss in hit 


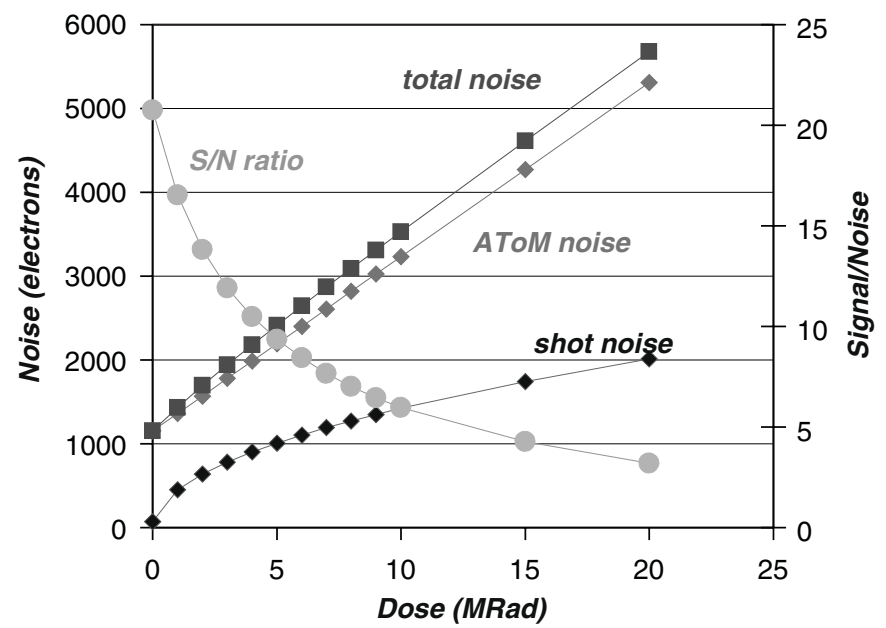

Fig. 2. Predicted noise levels from wafer (shot noise) and readout chip (AToM) and signal-to-noise ratio as a function of accumulated dose.

efficiency, when background hits precede real hits in the circular buffer, and a loss in resolution due to the deformation of clusters. Studies show that a significant impact is expected at occupancies above $20 \%$, with a loss of hit efficiency of $4 \%$ and a reduction in hit resolution of a factor two. Such occupancies will, however, only be reached in $10 \%$ of the layer 1 and 2 (midplane) channels, overlapping those most affected by radiation damage.

In summary, the SVT of BaBar has performed excellently in the first five years of operation, meeting design specifications in efficiency and resolution. We expect that in the next few years some small parts of the detector will be affected by an increase of noise on the readout chips due to higher than expected radiation doses. Due to sufficient redundancy in the design, this will only moderately affect the physics performance, and we therefore expect to successfully operate the SVT until the end of data taking.

\section{References}

[1] B. Aubert, et al., Nucl. Instr. and Meth. A 479 (2002) 1.

[2] V. Re, et al., IEEE Trans. Nucl. Sci. NS-49 (2002) 3284.

[3] V. Re, et al., Nucl. Instr. and Meth. A 409 (1998) 354.

[4] T.I. Meyer, Int. J. Mod. Phys. A 16S1C (2001) 1084.

[5] V. Re, et al., Nucl. Instr. and Meth. A 530 (2004) 7.

[6] S. Bettarini, et al., IEEE Trans. Nucl. Sci. 52 (2005) 1054.

[7] V. Re, et al., IEEE Trans. Nucl. Sci. 52 (2005) 787. 